SYMPOSIUM ON EVIDENCE BASED MEDICINE

\title{
Ethics, EBM, and hospital management
}

\author{
N Biller-Andorno, C Lenk, J Leititis
}

See end of article for

J Med Ethics 2004;30:136-140. doi: 10.1136/jme.2003.007161

authors' affiliations

.....................

Correspondence to:

$\mathrm{N}$ Biller-Andorno,

Department of Medical

Ethics and History of

Medicine

Georg-August-University

Goettingen, Germany;

nbiller@gwdg.de

Received

13 November 2003

Accepted for publication

27 November 2003

\begin{abstract}
Matters of hospital management do not figure prominently on the medical ethics agenda. However, management decisions that have to be taken in the area of hospital care are in fact riddled with ethical questions and do have significant impact on patients, staff members, and the community being served. In this decision making process evidence based medicine (EBM) plays an increasingly important role as a tool for rationalising as well as rationing health care resources. In this article, ethical issues of hospital management and the role of EBM will be explored, with particular reference to disease management programs, diagnosis related groups, and clinical pathways as recent developments in the German health care system.
\end{abstract}

W ithout doubt ethical aspects of hospital management need more attention and scrutiny than they have received up to now. Topics such as cloning, research on human stem cells, or preimplantation diagnosis have a much more immediate appeal as important moral issues than a field that is characterised by seemingly dry and technical terms such as "cost efficiency", "workflow management", "clinical pathways", "process orientation", and "total quality management". However, the tasks hospital managers are confronted with are in fact riddled with ethical questions. How to allocate available funds to departments, what portfolio of clinical services to offer, or how to assure a reliably high quality of services are matters that do indeed deserve ethical reflection and analysis. They need to be addressed directly and concretely, and not only by way of more general and abstract discussion on health care systems or access to health.

In this paper we want to spell out some of the ethical issues of hospital management. In particular, we will focus on the changes that are currently being brought about by the integration of evidence based medicine (EBM) into the hospital setting. In recent years EBM has been shown to be a powerful tool that can be used for various purposes and by different stakeholders in health care systems. Asking about the ethical implications of the introduction of EBM into hospital management can thus help to describe some of the major changes this area of medicine is undergoing at the moment, and at the same time contribute to an ethical analysis of EBM and the way it is implemented in practice.

\section{ETHICAL ISSUES IN HOSPITAL MANAGEMENT}

The specific social setting of the hospital abounds in ethical problems and conflicts that neither involve, nor are caused directly by, the hospital management. Rude behaviour towards patients during rounds, sloppy informed consent procedures before the inclusion of patients into clinical trials, or tensions within medical teams frequently do not even reach the management level. Nevertheless, hospital managers carry an exceptional responsibility for all activities in hospitals, because of their position in the institutional hierarchy and their decisive influence on the concrete framework within which the delivery of health care is taking place. Although it is less probable that decisions of the management will directly harm individual patients than a mistake by the medical staff, responsible decision making on the part of the hospital management is surely an important precondition if it is to be possible for hospital staff to integrate ethics into their daily work. The hospital manager bears responsibility to at least three parties: the patients who are being treated in the hospital; the staff; and the community that provides the funding for the institution. The longstanding guidelines on Ethical conduct and relationships for health care institutions of the American Hospital Association, (AHA) (first version issued in 1973), ${ }^{1}$ describe three corresponding areas of ethical concern: the responsibility of health care institutions for their patients; organisational issues within the hospital; and the community role of health care providers. These areas mirror the relevant interests which come together in the hospital: the interest of society to have effective health care institutions; the interest of patients to be treated adequately in the hospital; and the interest of hospital staff to work in an institution with appropriate ethical and social standards.

What do these three areas mean in the context of everyday decisions that have to be taken by hospital managers? As far as the first area is concerned, health care institutions have a special accountability to their patients, due to the particular nature of the relationship between patients and health care providers in the hospital setting, which is usually highly asymmetrical, in terms of knowledge and authority. To protect the patients' personal rights, the AHA's code of conduct mentions several instruments, including: informed consent for diagnosis and therapy; the use of advance directives; confidentiality regarding patients' private data; and respect for social, spiritual, and cultural needs and beliefs. In addition, hospital managements have to ensure continuity of health care for all patients; and at the same time they should enable doctors to offer patients possible treatment alternatives and engage in the improvement of the quality of care and therapy.

As for the second area, organisational and staff issues, the hospital management has to make sure the institutional mission statements and policies do not conflict with the professional ethical codes of-for example-doctors and nurses. Beyond this requirement, hospital managements should aim to encourage and promote initiatives from 
medical staff to implement ethical guidelines in their daily work. Anyone familiar with the impact of rationing in medicine knows, however, that the scarcity of resources for health care produces ethical problems-either of resource allocation, or of a lack of working hours, which may limit staff engagement in activities that are ethically important. Hospital management can use its influence to reduce such problems and to maintain ethical standards. As in any other enterprise, employees and medical staff deserve fair compensation, fair benefits, and the opportunity to take part in continuing education in order to steadily improve the quality of health care.

As far as the third area is concerned, the community role of health care providers requires hospitals to structure their health care provision so as to adequately meet the needs of the society in a specific local setting, in order to fulfil their public functions. Given that there are no clear upper limits to the demand and supply of medical goods as illustrated-for example, by the much discussed definition of health offered by the World Health Organisation (WHO) - it is necessary to use some rational criteria for resource allocation. Health care institutions must also give special consideration to the needs and medical problems of vulnerable groups, whether their vulnerability results from a specific lack of autonomy (as in the case of the very young, the elderly, or psychiatric patients), or from social marginalisation, as in the case of poor or inadequately insured people. Finally, health care institutions are financed by the fees of the insured or by other kinds of public funding and have therefore special duties to make "fair and effective use of available health care delivery resources". ${ }^{1}$ Hospital management is responsible to the public, at least in cases when it is the public which makes possible the existence and running of hospitals.

In sum, hospital managers can exercise influence aimed at promoting ethical health care over a wide range of organisational features. Although medical ethics mainly deals with conflicts between the patients and their health care providers, it is clear to us that the features which frame the institutional setting, such as the hospital, in which such conflicts take place, are of considerable importance as well for finding appropriate solutions. The emergence and formulation of common and consensual codes of conduct in many hospitals today can be interpreted as a sign of a growing consciousness of such a wider perception of factors shaping moral conflicts in medicine.

ON THE ROLE OF EBM IN HOSPITAL MANAGEMENT The tasks of hospital management can be described as pertaining to two large areas: quality management and allocation of resources. These are exactly the two areas where EBM has been put to use. ${ }^{23}$ EBM thus seems to be an ideal tool for decision making processes in hospital management. Given this background, it is surprising that so far the role of EBM in the hospital setting has been less discussed than its application in other fields of health care delivery and policy making, such as reimbursement practices by health insurance companies or the conduct of health technology assessments. One possible explanation for this is that EBM is still a relatively new movement, and in some countries is only now becoming an integral part of the definition of health care standards, including those of the hospital. ${ }^{4}$ Clearly, however, EBM has the potential to play an increasingly important role in the shaping and structuring of hospital health care. Therefore it is crucial to observe and critically analyse the implementation of EBM now, as it is taking place in the hospital setting, in order to anticipate and try to avoid ethical pitfalls as further developments occur.

The hospital management's position is extremely sensitive. Its multiple responsibilities open up the possibility for numerous conflicts between the various tasks-for example, between the need to respect the medical staff's autonomy in therapeutic decisions and the need for efficient use of the funding provided by the community. In addition, there is a large potential for conflict between the hospital management and other stakeholders, particularly physicians, policy makers, insurers, and the pharmaceutical industry.

What role can EBM play in such conflicts? In a tertiary care setting, for example, EBM could be used by the director of the oncology department as an argument to persuade the hospital management to allow more funding for the introduction of a costly new therapy, which has been shown to increase survival time for metastasised breast cancer. On the other hand, where there is no proof of the effectiveness of a therapy, the hospital management could cite the lack of an appropriate evidence base as a reason for the hospital to refuse to provide that therapy. It could also be used to weed out effective, but relatively costly, therapeutic options. And finally, EBM could be used by insurance companies to refuse reimbursement for medical services which have a low proven effectiveness, but are in high demand by patients-for example, an alternative medicine approach.

Just how and for whose benefits EBM is being employed and with what ethical implications can be understood only within the concrete context of a health care setting. ${ }^{5}$ In the next part of the paper, the German hospital care situation will be used as an example to illustrate possible forms of interaction between EBM and hospital management.

\section{DISEASE MANAGEMENT PROGRAMMES, DIAGNOSIS RELATED GROUPS, AND CLINICAL PATHWAYS - DEVELOPMENTS IN THE GERMAN HOSPITAL SYSTEM}

In order to explore the use of EBM in current developments in hospital management, the case of Germany could be of particular interest because major changes-which some have called the greatest since Bismarck's social security act in the 19th century-are currently under way. ${ }^{67}$

In Germany, quality management and efficient use of health care resources are required by book $V$, (Sozialgesetzbuch V, or SGB V) of the social security code, the relevant statute book for the regulation of benefits and the organisation of health care under the obligatory health insurance system. $\$ 92$ (directives of the federal board) of SGB V, for example, requires the Federal Board of Physicians and Health Insurance Agencies (Bundesausschuss der Ärzte und Krankenkassen) to formulate the directives (Richtlinien) necessary to assure the sufficient, suitable, and efficient provision of medical services that should be covered by public health insurances. §135(a) (obligation to secure the quality of health care) requires hospitals to participate in external measures for quality assurance in health care and to establish total quality management to improve treatment outcomes.

Since the year 2000, EBM has explicitly been introduced into this code. $\$ 137$ (e) ties evidence based guidelines into the German legislation as part of a project to reform and improve the public health system. For this purpose, a coordination committee has been established which has the task of selecting each year at least 10, mostly chronic, diseases for which there is unsatisfactory medical provision, in order to improve the quality of health care according to evidence based guidelines for these targeted diseases. The aim is to replace costly hospital treatments with standardised ambulatory treatment programmes, using specially trained physicians. These so called disease management programmes (DMPs) are now being established for diabetes, coronary heart disease, cardiac insufficiency, arterial hypertension, bronchial asthma, and chronic obstructive pulmonary disease. The basic idea is that health insurance companies put 
aside a certain amount of money to systematically improve the quality of medical provision for certain diseases, where, according to epidemiological data, the range of therapeutic options has so far been insufficiently explored. ${ }^{8}$ Health insurance companies will also have an economic benefit by offering disease management programmes to their membership, because money will be shifted to insurance companies with DMPs for chronically ill patients, from other health insurance companies without DMPs or health insurance companies with fewer chronically ill members. Thus health policy makers hope to give insurance companies incentives to compete for chronically ill members. As was expected, the introduction of these programmes has triggered a substantial debate between physicians, patients' advocates, and insurance companies. The introduction of these programmes means that third parties-that is, the insurers-will gain considerable power in determining medical care for patients who are covered by these programmes. Family doctors will act as "disease managers" and gatekeepers for the chronically ill patients, and will probably reduce their access to tertiary health care-that is, treatment and medical advice in highly specialised hospitals. It remains to be seen how EBM will be employed in the development of these programmeswhether it will be in an impartial or a selective manner.

Another important change concerns hospital payment systems, which will be changed from a fee for hospital day to a fee for a case, a reimbursement system based on the principle: "same prize for same product". The Australian ARDRG system (Australian refined diagnosis related groups system) is to be used as the basis for calculating hospital budgets. Under very great time pressure, this system is currently scheduled to be adapted to German conditions in a period of time so short as to be unprecedented. Hospitals could have started on 1 January 2003 on a voluntary basis, but all must start in 2004. After an adaptation period of only three years, this system will come into full economic use. ${ }^{9}$

In a peculiarly perfectionist manner, the Australian DRGs are being converted to German DRGs. Even though Germany has no experience with this payment system, it is the first country in the world to plan to calculate hospital budgets completely on a DRG basis. No exceptions are allowed for the special features and profiles of the different hospital services. Tertiary hospitals with a high level of medical services, especially university hospitals and emergency departments with intensive care units, are faced with the likelihood of significant budget reductions due to a high percentage of cost intensive medical treatments. This could have a negative impact on both the level of medical services and/or on the extent of innovation. In this situation the following factors are discussed as being significant for a hospital's survival: optimal documentation of diagnoses and procedures by physicians; high quality of services and products; minimal duration of hospital stays; a product portfolio defined by economic aspects; and, in particular, efficiency in resource utilisation.

One possible way to optimise resource utilisation is to define clinical pathways. These should, as some authors suggested, be evidence based. ${ }^{10}$ Given, however, that the minimum quality standards for medical services for any of the 800 DRGs are not currently defined, there is significant concern that economic aspects may play a greater role than the conclusions of EBM, at least during the system's introductory period. All hospitals will seek the best way to survive economically. Some health insurance companies, too, seem to be more concerned with defining groups of diseases not to be treated in a hospital than with setting quality standards-for example, by participating in therapy optimisation studies. Eventually the critical observation that EBM may be used by some interest groups as an opportunity to cut costs rather than as an instrument to enhance quality standards in health care may be proved correct. ${ }^{11}$

Another obstacle to the introduction of clinical pathways in hospital medicine is that workflow management as an effective instrument to increase quality and efficiency is met with scepticism by physicians. Many of them hesitate to participate in working groups which could define such processes. The high daily workload may be one reason. In addition, many physicians still regard medicine to be a special "art", which hinders the acceptance of standardised and fixed regulations in the medical treatment process. ${ }^{12}$ The more than 1300 published German official medical guidelines ${ }^{13}$ usually assure so called medical freedom, and most of these guidelines contain numerous diagnostic and therapeutic options that often lack scientific validation.

Such general medical guidelines are not, however, suitable for increasing efficiency in a hospital or a hospital department. Clinical pathways have to be defined for the disease groups having the greatest economic impact on a department. These pathways must include regulations for the complete hospital path of a patient. They should not only optimise the processes in one department, but also take into account the necessary actions of other departments and professions involved in the treatment process. They should meet the requirements of workflow management and be supported by adequate data processing. Ideally these pathways would be evidence based clinical practice guidelines, whose consistency could be reviewed according to the usual criteria: validity; strength of evidence and estimated outcomes; reliability/reproducibility; clinical applicability; clinical flexibility; clarity; and multidisciplinary process, scheduled review, and documentation. ${ }^{14}$ Unfortunately it can be expected that the implementation of such clinical pathways will probably happen not so much in synergy, but rather in confrontation with the medical staff.

\section{ETHICAL IMPLICATIONS OF THE USE OF EBM IN HOSPITAL MANAGEMENT}

The developments described above are characterised by the following main features: economic pressure; competition between hospitals; the growing influence of insurance companies and the predominant concern with economic over quality aspects as demonstrated by some of them; and the resistance of the medical staff to stringent guidelines that curtail their therapeutic freedom. From this background it seems likely that EBM will be employed in a confrontational manner by various antagonists, and not in an ideal, harmonious health care system, as is sometimes projected when the advantages of EBM are portrayed. The following applications can be projected. First, EBM will most likely be used for the purposes of rationalising as well as rationing resources, by policy makers, insurance companies, and also by hospital managers who have to see that their institutions remain competitive. An example of cost cutting with reference to EBM is the strategy of the German Red Cross hospital in Berlin in 1997. The hospital management were instructed to reduce costs by $10 \%$. According to a report from two participating hospital managers, this goal was achieved via economic side effects of the implementation of EBM into medical practice, such as standard operating procedures and clinical pathways. ${ }^{15}$ Secondly, EBM will rather be used to block than to encourage innovative technologies. Evidence for the clinical efficacy of new therapeutic and diagnostic procedures can only be produced by large, randomised, double blind multicentre studies, which take a great deal of time. In the meantime, health insurance companies deny the reimbursement of costs, because from their point of view any lack of evidence is a sign of missing efficiency or even of the futility of a medical intervention. In other medical areas-for 
example, oncology-cost reimbursement in Germany is partly denied because the number of treated patients for a new innovative therapeutic procedure is too small to reach high levels of statistical evidence. In the end, hospitals have to subsidise such patients from other financial sources or refuse to approve oncologists' requests to carry out these treatments. Thirdly, the management of an increasing group of patients will be predetermined by disease management programmes; the reimbursement of their care will be the basis of the corresponding diagnosis related group. It is likely that therapy will become more streamlined, a process that will be endorsed by stringent guidelines, probably to the dismay of clinicians and patients.

From a medical ethics point of view these developments raise a number of important issues that will merit the attention of policy makers as well as of all potential patients-that is, society as a whole.

- Which patient groups will end up worse off in their medical care because of EBM oriented therapeutic priorities?

- Will the development of disease management programmes, diagnosis related groups, and clinical pathways be compatible with physicians' therapeutic freedom, which is enshrined in many professional codes?

- How will hospital managers deal with therapeutic procedures that do not have a strong evidence base? Will they not be forced to cut them, given the economic pressures behind them? And how will it then be possible to take patient preferences into account-a matter that defenders of EBM frequently emphasise as its genuine goal. ${ }^{16}$

- Will most patients still have access to innovations in medicine?

- Will the pharmaceutical industry and related companies take advantage of the bias towards evidence based interventions and influence the therapeutic portfolio by a targeted production of evidence?

The answers to these questions will at least in part have to be based on conceptual deliberations as well as empirical data. It can be anticipated, for example that the methodological standards of EBM, in particular the so called gold standard of randomised controlled trials, will disadvantage areas of medicine such as psychotherapy and promote biotechnical and pharmacological interventions. ${ }^{17}$ This may lead to selective discrimination against mentally ill patients regarding their access to health care. These concerns need to be substantiated by further empirical work. Other projects, such as the introduction of DRGs for the reimbursement of medical services, have led in other countries, as in the case of US Medicare patients, to problematic ethical outcomes at the level of microallocation as well as at the level of medical infrastructure. The duration of hospital stay decreased, for example while the number of patients discharged in an unstable or sick condition increased. ${ }^{18}$ Whereas hospital physicians' professional autonomy may be curtailed by the introduction of DRGs, more visible competition between hospitals, allowing for a better informed comparison of performance, may on the other hand increase patient choice. The introduction of EBM in the context of the recent structural changes in the hospital setting is thus an experiment that should be accompanied by medical ethics through the use of prospective studies. The consequences of the experiment are of considerable importance. It would be an error to mistake these questions for simple technical, administrative details.

\section{CONCLUSION}

The tasks and responsibilities of hospital management contain many urgent ethical questions and problems-for example, who will decide in the future what is medically necessary and what a society can afford to pay? Many people have vague fears that medicine will develop to the point where physicians have to choose between the Nobel prize and the business prize. So far, however, there has been no adequate professional discussion of these issues.

Even more today than in the past, achieving the goals of equitable access, responsible cost containment, and effective quality assurance will require ethical decision making-as is the case with the patient physician relationship. California's medical board has set clear standards for physician conduct which could be regarded as significant for everyone working under economically driven tendencies in health policy:

The relationship between a physician and a patient must be inviolable. Included among the elements of such a relationship of trust are open and honest communication, including disclosure of all information necessary for the patient to be an informed participant in her/his care. This relationship is not to be constrained or adversely affected by any consideration other than what is best for the patient. The existence of other considerations, including financial or contractual concerns, is, and must be, secondary to the fundamental relationship. ${ }^{19}$

It might be of help if established ethical codes like the AHA code explicitly acknowledged and addressed ethical challenges in hospital management that are due to the increasing use of EBM for explicit or implicit economic purposes.

Will EBM be able to realise its positive potential as a useful instrument for quality management and resource allocation, or will it be used as a profit oriented "tool for excising large areas of health care expenditure" $?^{20}$ Unless EBM is used in a context characterised by transparency and openness to critical inquiries, and unless stakeholders resist the temptation to abuse EBM under the pretext of a value neutral, objective method for their own purposes, EBM's early critics might be proved right.

\section{Authors' affiliations}

J Leititis, Board of Directors, University Hospital, Goettingen, Germany N Biller-Andorno, C Lenk, Department of Medical Ethics and History of Medicine, Georg-August-University, Goettingen, Germany

\section{REFERENCES}

1 American Hospital Association (AHA). AHA management advisory-ethical conduct for health care institutions. Chicago: AHA, 1992. http:// www.hospitalconnect.com/aha/resource_center/resource/ resource_ethics.html (accessed 21 Jan 2004).

2 Dickenson D, Vineis P. Evidence based medicine and quality of care. Health Care Anal 2002;10:243-59.

3 Biller-Andorno N, Lie R, ter Meulen R. Evidence based medicine as an instrument for rational health policy. Health Care Anal 2002;10:261-75.

4 Biller-Andorno N, Karageorgiou A. Evidence based medicine in Germany. Paper presented at the European Union EVIBASE project State of the Art meeting. EVIBASE: Ethical Issues of Evidence Based Practice in Medical Care and Health Care; 2001 Feb 2-3; Maastricht.

5 Biller-Andorno N. Evidenz-basierte Gesundheitsversorgung-kritische Nachfragen vor medizinethischem Hintergrund. Wien Med Wochenschr 2002;152:161-4.

6 Leititis JU. Stationäre Leistungserbringung unter DRGs. Krankenhaus 2000; 11:897-902.

7 Leititis JU. Evidence for health policy: evidence based medicine/health care and the cost effectiveness debate. Commentary presented at the EVIBASE Workshop: Evidence based medicine as an instrument for rational health policy; 2001 Sept 28-29; Goettingen.

8 Glaeske G. Evidence based medicine aus Sicht der Krankenkassen-ein Rahmen für qualifizierte Therapiefreiheit und verbesserte Wirtschaftlichkeit? Z Arztl Fortbild Qualitätssich 1999;93:421-6. 
9 Roeder N, Schick J. Vorbereitung der deutschen Krankenhäuser auf die GDRG-Einführung. Ergebnisse einer Umfrage der DRG-Research-Group, Universitätsklinikum. Krankenhaus 2002:139-44.

10 Mueller M. DRG und EBM-auf dem Weg zu einer rationaleren Medizin. http://medweb.uni-muenster.de/zbm/medinfo/2001/2_drg.html (accessed 16 Jan 2004).

11 Hunter DJ. The practice of rationing health care in the United Kingdom. In: Breyer F, Kliemt H, Thiele F, eds. Rationing in medicine. Ethical, legal and practical aspects. Berlin: Springer 2002:39-52, at 43-4.

12 Praetorius F, Sahm S. Ethische Aspekte der Regularisierung ärztlichen Handelns. Ethik in der Medizin 2001;13:221-42.

$13 \mathrm{http}: / /$ www.uni-duesseldorf.de/WWW/AWMF/11/index.html laccessed 14 Jan 2004).

14 Field MJ, Lohr KN, eds. Clinical practice guidelines-directions for a new program. Washington, DC: National Academy Press, 1990.
15 Kersting T, Sobhani B. Der Einsatz von EBM erhöht die Qualität und hilft auch Kosten sparen. Erste Erfahrungen in den DRK-Kliniken Berlin. Führen und wirtschaften im Krankenhaus 1999;16:312-14.

16 Sackett DL, Rosenberg WMC, Gray JAM, et al. Evidence based medicine: what it is and what it isn't. BMJ 1996:312:71-2.

17 Lenk C, Biller-Andorno N. Evidenz-basierte Medizin und selektive Benachteiligung. In: Kettner M, Koslowski P, eds. Wirtschaftsethik in der Medizin: Wieviel Ökonomie ist gut für die Gesundheit? In press.

18 Kosecoff J, Kahn KL, Rogers WH, et al. Prospective payment system and impairment at discharge. The "quicker and sicker" story revisited. JAMA 1990;264:1980-3.

19 Medical Board of California. The physician/patient relationship. Sacramento: Medical Board of California, 1996.

20 Hope T. Evidence based medicine and ethics. J Med Ethics 1995;21:259-60. 